\title{
HYPERTELESCOPES: THE CHALLENGE OF DIRECT IMAGING AT HIGH RESOLUTION
}

\author{
A. Labeyrie ${ }^{1}$
}

\begin{abstract}
Sparse optical interferometric arrays of many apertures can produce direct images in the densified-pupil mode, also called "hypertelescope" mode. Pending the introduction of adaptive optics for cophasing, indirect images can also be reconstructed with speckle imaging techniques. But adaptive phasing is preferable, when a sufficiently bright guide star is available. Several wave sensing techniques, byproducts of those used on monolithic telescopes for some of them, are potentially usable. For cophased direct images of very faint sources in the absence of a natural guide star, a modified form of the Laser Guide Star techniques demonstrated on conventional and segmented telescopes is described. Preliminary testing in laboratory suggests further investigation. Recorded images, assumed co-phased, are also improvable post-detection with optical aperture-synthesis techniques such as Earth rotation synthesis, where data from successive exposures are combined incoherently. Nevertheless, the gain becomes modest if hundreds of sub-apertures are used. Image deconvolution techniques are also applicable, if suitably modified as demonstrated by Aime et al. (2012), and Mary (2012). Their modified deconvolution algorithms can extend the Direct Imaging Field (also called Clean Field) of hypertelescopes. More sub-apertures at given collecting area, implying that their size is reduced, improve the direct-imaging performance. The predictable trend thus favors systems combining hundreds of sub-apertures of modest size, if workable designs can be evolved. One such design, the "Ubaye Hypertelescope" entering the initial testing phase in the southern Alps, has a fixed spherical meta-mirror with a $57 \mathrm{~m}$ effective aperture, expandable to $200 \mathrm{~m}$. Preliminary results suggest that larger versions, whether spherical or active paraboloidal, can reach a kilometric aperture size at terrestrial sites having a suitable concave topography. In space, hypertelescope meta-apertures spanning up to $100000 \mathrm{~km}$ are in principle feasible in the form of a flotilla of mirrors, driven by micro-thrusters or by the radiation pressure of the Sun or lasers.
\end{abstract}

${ }^{1}$ Collège de France and Observatoire de la Côte d'Azur, 06460 Caussols, France 


\section{Introduction}

In the way of improved astronomical observation, the time has come for manyaperture optical interferometers providing direct high-resolution images. Most efficient perhaps will be the "hypertelescope" approach, using a sparse array of many sub-apertures and a densified pupil (Labeyrie 1996; Lardiere et al. 2007; Aime et al. 2012). Various opto-mechanical architectures and design concepts have been considered for versions on Earth and in space. Following the testing of a 3-mirror prototype with $9 \mathrm{~m}$ spacings at Haute-Provence (Le Coroller 2012), a larger instrument installed in the Ubaye range of the southern Alps has reached the testing stage (Labeyrie 2012). I describe some of the challenges raised by the direct and indirect production of hypertelescopic images, their phasing with adaptive optics and the observability of faint sources with a modified Laser Guide Star.

\section{The basic optics of hypertelescopes}

Among the various forms of optical interferometry which are considered for enhancing the resolution of astronomical observations, there had been some debate on the respective merits of pupil-plane vs. image plane arrangements. And on uniaxial vs. multi-axial devices. The hypertelescope concept involves a multi-axial beam combiner and densifier which can work either in the pupil or image planes, as well as through optical fibers where the notions of pupil and image vanish, as illustrated by Mourard et al. (2012) and Tarmoul et al. (2009). Pupil densification was already used by pioneer A.A. Michelson in his 20 feet interferometer at Mt Wilson, and the resulting intensification of the two-aperture fringe pattern on his retina, about $1600 \mathrm{x}$ at the full baseline setting, probably contributed to his observing success.

Since described in Labeyrie 1996, the hypertelescope principle has been discussed by different authors (Tarmoul 2009; Lardiere et al. 2007; Bouyeron et al. 2012; Patru et al. 2011; Aime et al. 2012). Various optical architectures can be adopted, one of which is a N-aperture Fizeau interferometer equipped with a pupil densifier, typically a small or even micro-optical accessory which can fit near the focal camera. Its effect is to shrink the diffractive envelope of the combined image and thus concentrate light into the central part of the interference function, thereby intensifying the image without affecting its pixel sampling. This is achieved at the expanse of the Direct Imaging Field of view, also called "Clean Field", which becomes shrunk down to $\lambda / s$, in terms of angular sky coverage, if $s$ is the minimal spacing of the sub-apertures. However, it now appears that suitably modified deconvolution techniques can retrieve in part the missing field (Mary, this volume).

At given collecting area, many small sub-pupils improve the imaging performance, with respect to fewer larger ones, both for direct imaging and for image reconstruction by aperture synthesis with a varying or rotating aperture pattern. The simple way of producing direct images which uses a Fizeau-type beam 
combiner, without pupil densification, does not exploit efficiently the exposures since much light is diffracted outside of the central interference peak in the spread function. The recorded pattern can be intensified by densifying the pupil, and considerably so if the aperture is highly diluted. In the absence of adaptive phasing, and in the use of "speckle interferometry" or "speckle imaging" for reconstructing the image as discussed in Section 2.2, such densification improves the signal/noise ratio and therefore the limiting magnitude. With adaptive phasing, generating a dominant peak in the N-aperture interference function, pupil densification is obviously beneficial for thresholded detectors, such as Michelson's retina and some infra-red cameras, since it can bring the level of interference peaks above the threshold.

Among the possible opto-mechanical design concepts for hypertelescopes, there are: a) arrays of telescopes having coudé foci, whether mirror-based or fibered, with optical delay lines feeding a beam-combiner; b) simplified designs resembling a giant sparse telescope, i.e. similar to the Arecibo radio-telescope although utilizing a sparse primary mirror, spherical and static, and not requiring delay lines since the moving focal combiner maintains the balance of all optical path lengths; c) Active versions of the latter ensuring a paraboloidal figure for the primary meta-mirror (Arnold et al. 1996). Terrestrial versions of types b and c, called Carlina, require a concave site for hosting the sparse mirror, and the absence of delay lines favors the use of numerous apertures, hundreds to thousands, having the potential of producing information-rich direct images. In space, versions as large as $100000 \mathrm{~km}$ are expected to become feasible in the form of a mirror flotilla. Laser-trapped versions are proposed.

\subsection{Imaging performance}

At radio wavelengths, interferometric arrays of antennas have achieved increasing success by using tens, hundreds, and thousands of elements. More than 10000 are now considered in some projects. A similar trend is also expected at optical wavelengths, following the science gains recently demonstrated with multi-telescope interferometers combining up to 6 beams (VLTI, CHARA...). These begin to produce reconstructed images, using aperture synthesis techniques analogous to those developed for radio interferometry. A basic difference, however, between the radio and optical versions of aperture synthesis currently utilized results from the incoherence of the optical data recorded sequentially at different times, with different aperture patterns. These optical exposures cannot be combined coherently for image reconstruction, since the phase distribution is missing. The incoherent combination gives a reconstructed spread function which is distorted and has a degraded dynamic range, compared to that provided by more sub-apertures, providing the same collecting area but simultaneously exploited for direct imaging. At radio wavelengths instead, the heterodyne detection can provide exposures shorter than the coherence time, and their complex amplitudes mapped at different times, with different aperture patterns, can be added for a coherent form of aperture synthesis providing a true synthetized aperture and the corresponding image. 
Conceivably, exposures as short as a pico- or femtosecond may become feasible at the combined focus of an optical interferometer, with an heterodyne beat to visualize the phase and thus obtain the complex amplitude map (Riaud 2012). Indeed, any incoherent source becomes spatially coherent if suitably filtered and observed with a sufficiently short exposure, shorter than the coherence time defined by the filter's bandwidth. Changing the aperture pattern and repeating the procedure would then provide a summed map of complex amplitudes, identical to that provided by the global aperture thus synthesized. And the intensity map which can be calculated is the object's image, convolved with the intensity spread function.

Although typically achieved at radio to sub-mm wavelengths, this has not yet been possible at wavelengths shorter than 10 microns. A reason is the very small number of photons per spatio-temporal field mode, at visible and near-infrared wavelengths, from usual astrophysical sources. If the collected starlight can be dispersed into a large number of narrow wavelength channels, each receiving a properly tuned heterodyne "clock" beam, and equipped with an ultra-fast detector, it could be attempted to analyze the data statistically to overcome the low photon count per mode. Whether this would theoretically improve the degraded form of aperture-synthesis process heretofore used at optical wavelengths remains to be explored. The practical implementation appears difficult.

It is therefore of interest to build efficient forms of many-aperture interferometers, forming a sparse meta-aperture much larger than feasible with a monolithic mirror or an "Extremely Large Telescope" (ELT) mosac mirror, the size of which is limited by the pointable supporting mount. On Earth, the size of such metaapertures may likely reach 1000 or $1200 \mathrm{~m}$ if built somewhat like the Arecibo radiotelescope within a natural depression. Larger versions, spanning perhaps $10 \mathrm{~km}$, can also be considered, but in the absence of sufficiently wide and deep depressions, long delay lines would be needed and their high cost may constrain the sub-aperture count. Also, the implementation of laser guide star systems, needed for cophased direct imaging on faint sources, may be more difficult with such delay lines and complex coudé trains.

In space, the technical challenge is very different, and baselines spanning $100000 \mathrm{~km}$ appear feasible at some stage (Labeyrie 2009). First-generation proposals for hypertelescope flotillas of mirrors have been submitted to the space agencies NASA and ESA (Labeyrie 2009). A low-cost version involving thousands of inch-sized mirrors, accurately controlled by a pair of laser beams forming a "standing wave trap", has also been conceived and subjected to laboratory tests in high vacuum (Labeyrie et al. 2010).

\subsection{Pending phasing: The speckle imaging mode of hypertelescopes}

Pending adaptive phasing, early science can be performed with a hypertelescope using speckle interferometry and speckle imaging with triple correlations (Lohmann et al. 1983; Tcherniavski et al. 2011). This has been simulated for the hypertelescope case by Surya et al. (2012) who obtained encouraging results. In monolithic 
telescopes, higher limiting magnitudes, beyond $m_{v}=18$, have been achieved with speckle interferometry than with adaptive optics using a natural guide star, the magnitude of which rarely exceeds $m_{v}=13$. Pierre Riaud (private communication) suggests that the limiting magnitudes are in fact the same in both modes, a point which deserves verification. If speckle imaging proves more sensitive in hypertelescopes, it may remain of interest, even after adaptive cophasing becomes installed, on rather faint and simple objects not located within the isoplanatic patch of a brighter star. But Laser Guide Star systems, if they can be implemented on hypertelescopes for cophasing on faint sources (section below), should become preferable in all cases.

Does pupil densification improve the speckle interferometric signal/noise ratio and limiting magnitude? In monolithic telescopes, the photon-limited signalto-noise ratio in speckle interferometry is classically known to be $S / N=1 / 2$ $(1+k)^{-1} N_{p h s} N_{p}^{1 / 2} N_{s}^{1 / 2}$ (Dainty 1974), if $N_{p h s}$ is the number of star photons per speckle, $\mathrm{k}$ the number of photons from the sky background and $N_{p}$ the number of recorded short exposures. $N_{s}=\left(D / r_{0}\right)^{2}$ is the number of speckles per exposure, if $D$ is the aperture diameter and $r_{0}$ Fried's radius describing the size of phase cells. In a Fizeau interferometer having a meta-aperture of size $D_{m}$, containing $N_{a}$ sub-apertures of size $d$, here assumed to be distributed non-redundantly and to match the size $r_{0}$ of seeing cells, the speckle count within the image's diffractive envelope is now $N_{s a}=\left(D_{m} / r_{0}\right)^{2}=(D / d)^{2}=N_{s}\left(D_{m} / D\right)^{2}$. The speckle count thus increases quadratically with the array size $D_{m}$. Densifying the pupil by a factor $\gamma_{d}$ shrinks the speckle envelope in the same ratio, and therefore also decreases the number of speckles $N_{s}$ as $1 / \gamma_{d}^{2}$. Energy being conserved, the number of photons per speckle correspondingly increases, and eventually, at full densification, reaches that of a monolithic aperture having the same collecting area. According to Dainty's expression, pupil densification thus increases the signal/noise ratio, and matches that of a monolithic aperture having equivalent collecting area if also operated in the speckle interferometry mode. But it must be verified whether Dainty's expression remains applicable in the hypertelescope situation. In practice, pupil densification also relaxes the monochromaticity requirement, down to the monolithic value, thus also further enhancing the photon count per speckle $N_{p h s}$ and therefore the $S / N$ ratio, unless perhaps if many narrow wavelength channels can be simultaneously exploited in parallel by a photon-counting camera. The effect of densification on the signal/noise ratio of "speckle imaging" reconstructions with triple correlations of recorded images also deserves to be explored.

\subsection{Adaptive phasing}

Adaptive phasing is highly desirable when a guide star, whether natural or artificial, is available near the observed source. Commercial deformable mirrors such as Boston Micromachines' MEMs with tip-tilt-piston facets appear suitable and may be installed at the exit of the pupil densifier. The usual types of wave sensor, such as the Shack-Hartmann or curvature sensor, serving in conventional telescopes, however, are not suitable since the measurements of local slope or curvature errors 
in the wavefront assume its continuity to reconstruct it. Other methods which appear suitable are:

a) Hierarchical phasing (Pedretti \& Labeyrie 1999);

b) A modified version of the Shack-Hartmann method, with triplets of adjacent sub-apertures feeding each lenslet, with overlap, to provide polychromatic interference honeycombs from which phase maps can be derived (Cuevas 2007);

c) The dispersed-speckle method (Borkowski \& Labeyrie 2004; Martinache 2004), specifically developed for hypertelescopes;

d) The chromatic phase diversity method (Mourard et al. 2012);

e) The modified phase diversity method of Bouyeron et al. (2012) using a genetic algorithm.

Among these methods, b) is analogous to Shack-Hartmann and curvature sensing in the sense that it reconstructs the global map of piston errors from local slopes measured among clusters of adjacent sub- apertures. A difference, however, is that the local slope signal is derived from the position of polychromatic honeycomb-like interference patterns. The guide star should not be much resolved by the clusters of subapertures, but can be resolved by the global aperture. Methods a, c, d and e exploit interference speckles, which contain contributions from all baselines, short and long. They are therefore affected if the star is resolved by the latter.

\subsection{Principle of a "Hypertelescope Laser Guide Star" (H-LGS) system}

For observing faint sources, providing less than a few photons per seeing cell and spectral channel in exposures shorter than the turbulence lifetime, Laser Guide Stars have been successfully used with adaptive optics on monolithic or mosaic telescopes, and also provide the best hope of cophasing terrestrial hypertelescopes. If somewhat modified, as briefly suggested in Labeyrie (2008), the Laser Guide Star systems developed for monolithic apertures may also become suitable for the sparse apertures of hypertelescopes. As shown in Figure 1, Young's fringes can be projected by a sodium laser toward the sodium layer at $92 \mathrm{~km}$ altitude, using three or more apertures distributed like those of the hypertelescope, or actually belonging to its mirror array. Back-scattered light returning through the same apertures carries information on the cophasing errors.

The intensity spread functions of a multiple aperture for the up-going beam reaching the sodium layer and the down-going light back-scattered through the same multiple aperture are identical and similarly oriented if seen from below, while the down-going geometrical imaging of the spatially incoherent apparent Fizeau pattern in the sodium layer is inverted (Fig. 1). The double-pass pattern recorded by the camera is therefore a convolution of the single-pass Fizeau intensity pattern $I_{h}(x, y)$, projected at altitude $h_{s}$ within the sodium layer, with an inverted copy of itself $I_{h}(-x,-y)$.

$$
I(x, y)=I_{h}(x, y) \otimes I_{h}(-x-, y) .
$$




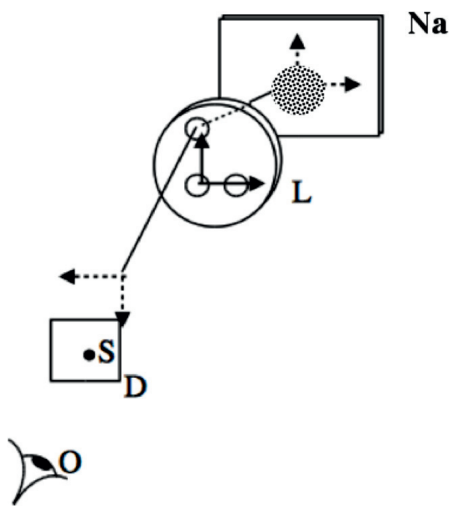

Fig. 1. Principle of H-LGS and coordinate symmetry. A sodium laser source S is projected by lens L, carrying a multi-aperture mask, toward the sodium layer $\mathrm{Na}$, where interference fringes are formed within the diffraction envelope. The resonantly scattered light is made incoherent by the fast motion of sodium atoms, and part of it propagates back through the sub-apertures toward S, where a camera (not shown) records the double-pass interference pattern. Arrows indicate the coordinate symmetry, affected by the inversion of the lens image.

Phase information can be extracted from the recorded fringe exposure by calculating its two-dimensional Fourier transform, which is the product of the pupil's complex autocorrelation with the inverted copy of it, which is also its complex conjugate. The product is therefore a real function, and its modulus is the square of the pupil's single-pass autocorrelation function modulus:

$$
\begin{aligned}
I(u, v) & =A C\left[P_{h}(u, v)\right] A C\left[P_{h}(-u,-v)\right] \\
& =A C\left[P_{h}(u, v)\right] \overline{A C}\left[P_{h}(u, v)\right] \\
& =\left|A C\left[P_{h}(u, v)\right]\right|^{2}
\end{aligned}
$$

where $P_{h}(u, v)$ describes the complex pupil carrying the single-pass phase error, and $\mathrm{AC}$ is the auto-correlation. If there are redundant baselines in the cluster of sub-apertures, each corresponding auto-correlation peak contains a sum of complex terms, and their modulus is consequently sensitive to any phase difference among these terms, thus allowing their measurement.

This method differs from those developed by Bonaccini (2004) and by Rabien et al. (2006) for reducing the LGS cone effect in a large telescope. The H-LGS principle is more related to the analysis of Mavroidis (1991), showing in a different context that retro-propagation through the same cluster of sub-apertures and seeing preserves phase information.

The typical fringe period in the sodium layer is of the order of $6 \mathrm{~mm}$, within a diffractive envelope spanning $500 \mathrm{~mm}$ if the emitting sub-apertures are $120 \mathrm{~mm}$ wide, assumed smaller than Fried's r0 value. If observed with temporal resolution 
longer than microseconds, the light resonantly backscattered by the sodium atoms appears to be spatially incoherent, owing to the Doppler spread induced by their fast Brownian motion. The pattern thus projected onto the sodium layer, fringed and serving as the artificial guide star, jitters in response to atmospheric phase shifts in the up-going beams. Nevertheless wave sensing in the returning beams remains possible with methods such as a) and b) mentioned in Section 2.3. Indeed, the incoherent fringed pattern, as seen in the sky through any cluster of adjacent sub-apertures in the hypertelescope array, gives a convolved image which retains appreciable fringe contrast, particularly if the aperture pattern is periodic or if the same cluster serves for the up- and down-going laser light. Also, the cluster should have more than three sub-apertures, so that at least one phase error can be calculated. The smallest type of cluster meeting these conditions is a quadruplet of sub-apertures, and they should preferably be adjacent to minimize anisoplanaticity effects.

If both wavelengths of the sodium doublet are emitted by the laser, and if the piston errors are pre-adjusted to be less than the doublet's coherence length, about 100 microns (instead of $100 \mathrm{~mm}$ for the Doppler-shifted back-scattered light from a single line), then piston errors can be derived and mapped for adaptive correction.

However, this is affected by the "cone effect", i.e. the propagation mismatch of light rays from the artificial star and the observed natural source, becoming particularly strong with a kilometric meta-aperture. To avoid it, each sub-aperture should capture back-scattered laser light which includes rays co-propagating with those from the observed source, thus crossing the same atmospheric turbules which affect their phase. The condition can be met by using in parallel many such artificial guide stars, arrayed within the sodium layer as a projection of the hypertelescope's aperture pattern toward the observed celestial source. If Fried's radius $r_{0}$ and the sub-aperture size $d$ are such that $r_{0}=d=25 \mathrm{~cm}$, then the size of the projected Airy spots in the sodium layer matches that of the sub-apertures. Starlight crossing the sodium layer through these laser spots enters the corresponding subapertures, together with some of the back-scattered laser rays which follow the same path through the atmosphere and are thus affected by the same turbulence. In addition, since each laser-illuminated spot in the sodium layer must contain interference fringes, it must also receive coherent laser light from a few other subapertures.

This is achievable if their common laser source is a point source located some distance above the science camera, suspended in the focal plane of the metamirror, so that it be imaged by it at the sodium layer. As sketched in Figure 2, the arrangement is parallelized by installing an array of such laser sources, each illuminating a cluster of sub-apertures which projects a separate laser spot into the sodium layer.

For connecting the local phases thus obtained and deriving a global map of the starlight phases at all sub-apertures, the clusters have to contain at least four sub-apertures and to be partially overlapping. The map calculation has to assume that the atmosphere's isoplanatic angle $i_{0}$ is larger than the apparent spacing $s / h_{s}$ 


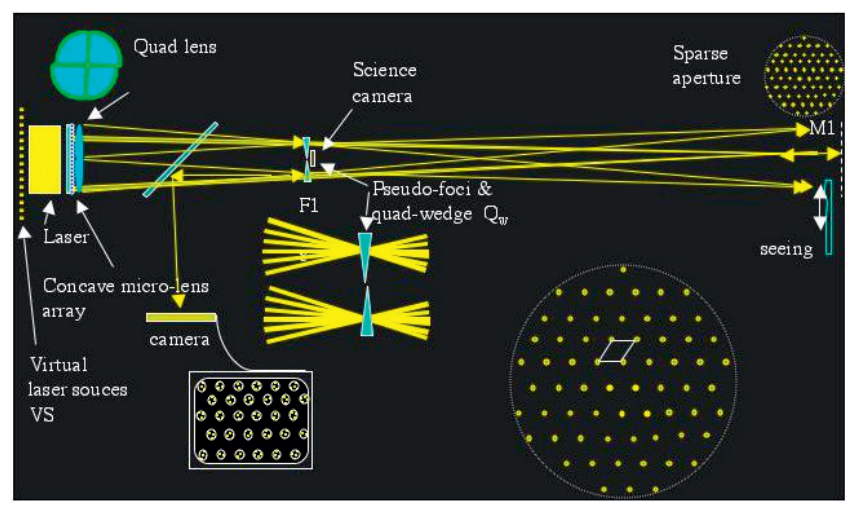

Fig. 2. Example of Hypertelescope Laser Guide Star system: segment M1i of the metamirror M1 is focusing onto the sodium layer (not shown, as it would be located far at left on a scale drawing) the image S'i of a point source Si of laser light, located slightly above F1, the focal image of the observed star. The exact transverse position of segment M1i is such that the reflected laser ray is directed toward the observed star, thus following in the opposite direction the propagation path of its ray reaching the segment. The same laser source Si also illuminates at least three additional M1 segments, which focus the corresponding beams at the same S'i position, thus generating interference fringes within the diffractive envelope in the sodium layer. The arrangement is parallelized with additional laser sources, projecting into the sodium layer an array of fringed spots. The array pattern is identical to M1's sub-aperture pattern, and the clusters of M1 elements focusing each S'i spot are partially overlapping so that the measured fringe phases be propagated for building a global map of phase errors affecting the observed source's image. The multiple laser source, where each laser illuminates a single cluster of M1 segments, is here implemented with a single laser illuminating a concave micro-lens array, next to a group of four or more adjacent lenses and facing wedges.

of the laser spots in the sodium layer, if $s$ is the sub-aperture spacing, matching the laser spot spacing, and $h_{s}$ the altitude of the sodium layer. The spacing matching the condition is then $s=h_{s} i_{0}$, typically amounting to $10 \mathrm{~m}$ with $20^{\prime \prime}$ isoplanatism, and then implying $N_{a}=10000$ sub-apertures within a kilometric meta-aperture. With such large numbers, the needed laser power may become a practical challenge since a few tens of watts are needed per sub-aperture.

I made a simple laboratory experiment, sketched in Figure 3, to verify the phase sensing scheme with a single cluster of sub-apertures. A laser source is focused toward a rotating reflective diffuser, simulating the sodium layer, through a multiple aperture. The pattern of backscattered light returning through the same multiple aperture and lens is recorded by a camera, virtually located in the plane of the laser source, but separated by a beam splitter. Rotating the diffuser simulates the motion of sodium atoms in the atmospheric sodium layer, thus smoothing the speckled backscattered light reaching the aperture mask and causing the apparent 


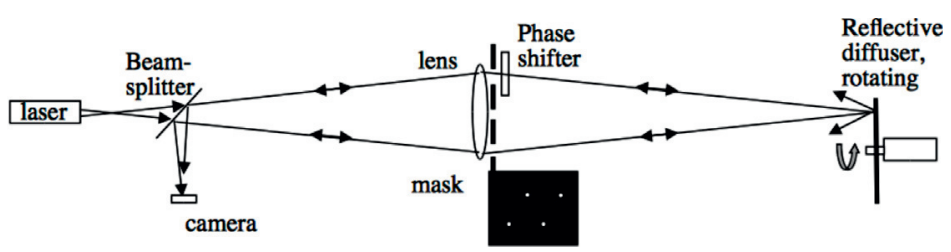

Fig. 3. Laboratory simulator of the H-LGS scheme. Laser light is focused toward a pinhole, itself relayed by a lens toward a reflective diffuser, which can be rotated to simulate the motion of sodium atoms in the atmospheric sodium layer. A multi-aperture mask inserted near the lens simulates an array of hypertelescope sub-apertures, and a cluster such as the shown rhombus can be selected with a second mask. A bumpy glass plate near the sub-apertures produces phase errors. The back-scattered light returning from the diffuser and through the same mask is recorded by a camera, through a beamsplitter.

fringe pattern on the rotating diffuser to become spatially incoherent, which reduces somewhat the fringe contrast in the recorded long exposures. Phase errors, introduced on sub-apertures by a distorted glass plate, are measured by Fourier analyzing the recorded fringe patterns.

Figure 4 shows the recorded exposures. Appreciable contrast is retained in the fringe patterns when the diffuser is rotated. The patterns are seen to be influenced by the phase error, as it is also apparent in their calculated Fourier transforms by comparing the intensities of the four median peaks, each involving a pair of redundant baselines.

\subsection{Phase determination with overlapping clusters of four subapertures}

The laboratory simulation has confirmed that the recorded fringe pattern is influenced by phase errors, using either a rhombus-shaped cluster of four adjacent sub-apertures among a triangular array, or a centered-hexagon cluster of $6+1$ subapertures. Further testing in the laboratory is undertaken by Paul D. Nuñez for a more realistic simulation and hardware development, toward some real testing envisaged with the "Ubaye Hypertelescope" prototype and the development of a working system. Its optical scheme, providing from a single laser source the required multiplicity of laser beams, is sketched in (Fig. 2). The design can be downsized for laboratory simulations.

The laboratory simulation is complemented by numerical simulations and data analysis that will quantify the accuracy of the phase determination accross the array in the presence of non-uniform illumination in the sub-apertures, photon noise, etc. Paul D. Nuñez has used the formalism described in the preceeding section to perform numerical simulations of similar data to that presented in Figure 4. An example is presented for illustrative purposes in Figure 5. The top and bottom set of images differ from each other by a phase difference introduced in one of the four sub-apertures. 


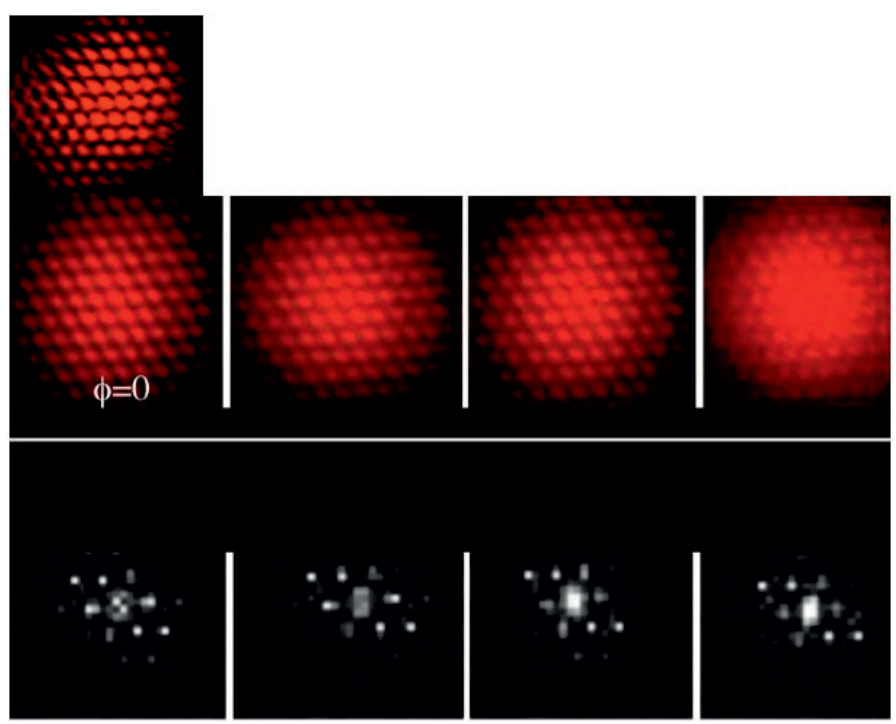

Fig. 4. Exposures obtained with the rhombus cluster of four sub-apertures and different phases errors. The top left exposure involves a static diffuser and no phase error. The middle row involves the rotating diffuser and shows the effect of various phase errors on the long-exposure pattern. The bottom row shows the corresponding Fourier transforms, where the median peaks generated by each redundant pair of baselines have their intensities influenced by the phase errors.

\subsubsection{Deriving the phase errors}

With a rhombus cluster of sub-apertures, three of the phase values are known or can be taken as zero if image motion is accepted, and the fourth has the unknown value $\phi_{4}$. In the complex autocorrelation of the pupil, the peak related to baselines 2-3 and 1-4, for example, has a value $1+e^{i \phi_{4}}$, and its square modulus $I_{14}=$ $\left(1+e^{i \phi_{4}}\right)\left(1+e^{-i \phi_{4}}\right)=2\left(1+\cos \left(\phi_{4}\right)\right)$ is the modulus of the corresponding peak observed in the calculated Fourier transform of the camera image. Its value varies from 0 to 4 . The central peak's modulus is $I_{0}=4^{2}=16$. The unknown phase $\phi_{4}$ is thus determined as: $\phi_{4}= \pm \arccos \left(I_{14} / 2-1\right)= \pm \arccos \left(8 I_{14} / I_{0}-1\right)$.

The sign ambiguity is resolvable by trial and error, in one or two steps of piston correction, or by a phase diversity method, using a second camera exposure with a known amount of defocus, achieved by range-gating laser pulses to select backscattered light from a different sub-layer of the sodium layer. Pierre Riaud suggests to record a second image separated with a beam splitter, and where a known phase shift is added. For increased sensitivity, many such layers can be simultaneously observed in separate temporal channels. More than four apertures can presumably be used in a cluster, although the phase extraction is more elaborate with more redundancy, i.e. if more than two baselines contribute to each Fourier peak, as it is the case for a centered hexagon. 

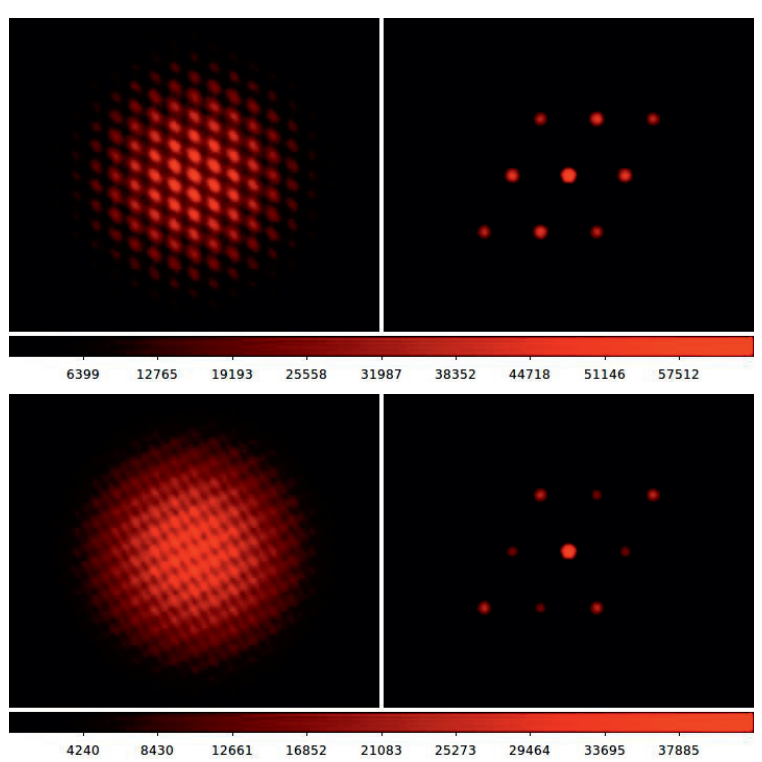

Fig. 5. Numerical simulation, made by Paul D. Nuñez, of fringes (left) and their Fourier transform (right, and in square root scale for better visibility) as would be obtained with a laser guide star. These correspond to four sub-apertures arranged in a rhombus pattern. The two sets of images differ by introducing a phase delay of $P I / 2$ in one of the sub-apertures.

\subsection{Deconvolution of phased images}

Image deconvolution has been fruitfully used in different situations of astronomical imaging, particularly with radio-interferometers and other situations where the spread function has numerous and strong sidelobes, as is the case with interferometers containing few apertures, or when dealing with highly contrasted sources, such as a star and its planets. Aime et al. (2012) and Mary (this volume) have began exploring the case of hypertelescope images. Because of the pseudo-convolution which accounts for image formation in this case, they had to modify the established deconvolution methods and obtained encouraging results. An unexpected gain has been the retrieval of sources located outside of the "Direct Imaging Field", also called "Clean Field". Further work toward characterizing the potential of these methods will be beneficial for assessing the science program of large hypertelescopes (section below). Like in the case of speckle imaging (section above), the effect of the pupil densification factor on the signal/noise ratio, using detectors which are photon-limited or not, should also be analyzed.

Another aspect that can be studied with deconvolution is the dynamic range. The array configuration has an effect on the dynamic range of the direct image (Lardiére et al. 2007), and some configurations may be better than others in this respect. However, it is not known if this will remain true in the deconvolved images. This is one of the issues that will start to be addressed in an upcoming 
paper that deconvolves simulated images of Betelgeuse (Patru et al. 2013 in prep.). Paul D. Nuñez, in collaboration with David Mary, Fabien Patru and Claude Aime are making a more systematic study of these questions.

\section{Construction of the "Ubaye Hypertelescope" prototype}

Following more than a decade of analysis and simulation by various authors, together with laboratory testing, the direct-imaging performance and sensitivity gain foreseen for hypertelescopes has prompted our group to build two prototype versions of a Carlina hypertelescope: 1) a first prototype at Haute-Provence observatory, utilizing a tethered balloon to carry the focal optics and camera $35 \mathrm{~m}$ above a triplet of mirrors spaced $9 \mathrm{~m}$ apart (LeCoroller et al. 2012); 2) and then the larger "Ubaye hypertelescope" prototype utilizing a suspending cable, stretched across a deep valley in the Ubaye mountains of the southern Alps to carry a focal gondola $100 \mathrm{~m}$ above a $57 \mathrm{~m}$ meta-aperture, expandable to $200 \mathrm{~m}$ with potentially 100 or more mirror elements (Labeyrie et al. 2012).

\subsection{Opto-mechanical concept and design}

Among the various possible architectures for Earth-based hypertelescopes, flat or concave, the Arecibo-like concept involves a large fixed and spherical concave metamirror focusing images toward one or more gondolas driven along the focal surface. It does not require delay lines and thus favors the use of numerous sub-apertures, in the form of mirror segments sparsely arrayed across the large "meta-mirror". For a given collecting area, a large number of small apertures are preferred to fewer large ones, in accordance with the theoretical results indicating that the direct-imaging performance improves markedly with the number of sub-apertures. Such optomechanical designs are called "Carlina", name of a large alpine thistle flower which is stem-less and contains hundreds of smaller flowers within its "meta-flower".

As previously described (Labeyrie et al. 2012), the optical train of the "Ubaye hypertelescope" has a fixed, sparse and spherical M1 primary mirror. It also has a suspended focal gondola, accurately driven by six oblique tethers to track the star's image. It contains a spherical M2 mirror at the best focus within the caustic surface, a strongly aspheric M3 mirror located in the pupil plane, and additional lenses for densifying the pupil. An optional flat coudé folding mirror addresses a small collecting telescope located in the North-facing slope at the polar projection of M1's curvature center C1.

Following the optical design study by André Rondi, further developed by Rijuparna Chakraborthy, the concept has been also analyzed by Enmark et al. (2011) who included a detailed model of the gondola's drive, including the effect of wind disturbances. A price to be paid for the optical simplification of the Carlina design, with respect to hypertelescopes using delay lines, is the pupil drift occurring within the gondola as it tracks the star, while keeping its optical axis aligned with it. This can be accommodated by moving an optical element within the gondola, whether passively with a pendulum drive such as adopted for the 
Haute-Provence prototype, or actively with for example a motorized version of it. Another possibility is the use of a photo-activated pupil densifier driven by laser beams received from each M1 mirror. The latter putative type of integrated "pupil-tracking densifier" can use photo-active materials such as a thermoplastic/photoconductive film stack (Crandall et al. 1985) where the incident array of projected laser spots reversibly imprints micro-lenses, enabling thus to follow the drifting motion of the laser spot array. Variants with photo-refractive films may also be considered. Pupil densifier types using a single micro-lens array, as described in Pedretti et al. (1999), are particularly suited for incorporating such devices.

\subsection{Experience gained with "Ubaye Hypertelescope"}

As described in Labeyrie et al. (2012), the construction of the "Ubaye Hypertelescope" prototype since 2011 in the mountain valley at 2100-2300 m altitudes has raised unusual challenges, in comparison with those experienced when building interferometers of the previous generation. Both the optical and mechanical concept were simplified, but unexpected issues were met, such as the manipulation of long cables and their protection from avalanches and tree branches, the invasion by sheep flocks tangling their legs in wires, and the colonization of mirror supports by nesting wasps. Also, part of the site being within the Parc National du Mercantour, it has been important to protect birds from collisions with the cables (particularly the Black Grouse Lyurus tetrix and the large endangered Bearded Vulture Gypaetus barbatus). Some team members expressed fears of attacks by wolves which are present at the site.

For an optimal insertion of the M1 locus in the terrain topography, its mapping has been done with GPS/RTK techniques approaching decimetric accuracy by Loic Evrard and Marion Gaudon (Institut Geographique National), and pursued by Martine Roussel and Jerome Maillot, who also used a laser theodolite. Remi Prudhomme has designed and assembled the driving electronics and the extended wifi link between its elements, located together with the winches a few hundred meters apart. Denis Mourard has tested the system and written the high-level control code based on the spherical trigonometric model. He has been able to observe the focal gondola's stellar tracking motion with the coudé telescope and verify its accuracy, expected to reach $1 \mathrm{~mm}$ during typical good observing nights where wind velocity is low.

We have installed a pair of mirrors on stiff tripods, $16 \mathrm{~m}$ aprart, for assessing the system with fringes on a bright star. This has not yet been achieved, but no major problem has been identified. We have developed techniques for the optical alignment, including the acquisition of the coudé beam toward the $20 \mathrm{~cm}$ collecting telescope and the gondola's drifting attitude which must be controlled in addition to its drifting position.

With the heavy snow and difficult accessibility in wintertime, it is expected that remote observing will become possible at a later stage, without any human attendance. 


\subsection{Spherical or paraboloidal meta-mirror?}

The LOVLI concept for a Moon-based interferometric array proposed by Arnold et al. (1996) involved a sparse and active paraboloidal meta-mirror, the segments of which were controlled by actuators to keep the paraboloid axis pointed toward the source being tracked. Also, a larger variant of the Arecibo radiotelescope, the $500 \mathrm{~m}$ FAST radio-telescope becoming built in China, has an active paraboloidal mirror. Its shape is dynamically adjusted by actuators so as to remain paraboloidal while its axis direction tracks the observed source.

A paraboloidal design comparable to the LOVLI is now studied by Yves Bresson as a possible future upgrade for Ubaye Hypertelescope. His initial ray-tracing analysis with Zemax code indeed suggests that the spherical meta-mirror, once equipped with a few actuators on each segment, can be re-shaped into a paraboloid by computer commands in a matter of minutes and reversibly. The shifting also requires removing the focal corrector of spherical aberration needed in the spherical case.

The old controversy on the merits of paraboloidal and spherical telescopes, correctible by Schmidt plates or smaller elements near the focal plane, suggests that each mode has specific advantages and drawbacks. For example, the focal optics is simpler in the paraboloidal case, especially if there is no coma corrector. But, with a spherical M1, its static geometry and the multi-gondola option for simultaneous observations in widely separated fields may impact the cost and science output. Among the comparison elements deserving further study are: 1) the cost of active M1 segments, vs. that of spherical aberration correctors in multiple gondolas, and: 2) the field-of-view coverage achievable with coma correctors in the single focal gondola of a parabolodal meta-mirror, vs. that achievable in each gondola exploiting a spherical M1. In both cases, coma correction can be extended with local correctors within each field channel covering the diffraction lobe of the sub-aperture.

\subsection{Science capabilities}

In addition to stellar physics, with spatio-spectral direct imaging on resolved stars and their environment, a challenging related possibility is the production of transit images when an orbiting exo-planet crosses the star's apparent disk. Such displays should be reasonably contrasted if there are many apertures and if the planet is resolved or nearly so. They are potentially easier to observe than the presence of the same planets when not transiting. In the latter case, advanced coronagraphic techniques are needed to evidence the comparatively faint planet surface. In the limit case of a planet just entering transit, or emerging from it, bright refractive arcs are also likely observable, such as seen during the recent Venus transit across the Sun. This should provide valuable opportunities to probe spectroscopically the exoplanet's atmosphere thus sampled by the star's light at grazing incidence, as needed for searching bio-signature molecules (Leger et al. 2011).

But the large gain in limiting magnitude expected with hypertelescopes, especially when equipped with a Laser Guide Star, potentially brings a diversity of 
galactic and extra-galactic sources within observing reach: Active Galactic Nuclei and their fast-orbiting central stars giving information on the mass of a central dark hole, jet structures of galaxies or QSOs, and gravitational lenses.

\section{Feasibility of 1000-1200 m Carlina hypertelescopes on Earth}

The preliminary experience gathered while building Ubaye Hypertelescope already suggests that much larger versions can be built in larger and deeper Andean or Himalayan valleys having a suitable topography. The feasibility and the design options for a $1200 \mathrm{~m}$ "Extremely Large Hypertelescope" (ELHyT) have been discussed by Labeyrie et al. (2012). An H-LGS system such as discussed in Section 2.4 above is essential for fully exploiting the science potential of an ELHyT toward the faintest limiting magnitudes for observing cosmological sources. These limiting magnitudes can match in principle those accessible to an ELT of similar collecting area, and similarly equipped with a Laser Guide Star (Boccaletti et al. 2000). Together with the 100 microarc-second resolution attainable at visible wavelengths, a 30x gain with respect to a $40 \mathrm{~m}$ ELT, this announces major science inroads toward cosmology on the faintest known galaxies at the edge of the observable Universe.

The prospect deserves a comparative study of ELT and ELHyT technologies in terms of science, readiness and cost efficiency, which the funding institutions should initiate as part of their decision-making process. In the ELHyT case, features which may impact the compared cost are: 1) the absence of a pointable mount; 2) absence of a dome; 3) the smaller mirror elements, preferable for improved imaging performance at given collecting area, allowing a reduction of the glass thickness; 4) the resulting use of lower-grade glass such as Pyrex, becoming adequate for the smaller mirrors; 5) the high-yield mirror figuring techniques then also becoming available; 6) the risk reduction achievable by deploying and testing a few mirror elements with a focal gondola and its tracking system; 7) the progressive construction and early science achievable, as demonstrated with the ALMA; 8) the availability of the numerical model of gondola control and dynamic behaviour, developed by Enmark et al. (2011); 9) the existence of an access road at some of the mountain sites considered, such as Spiti valley (India, at $4000 \mathrm{~m}$ altitude).

\section{Hypertelescopes in space}

Large flotillas of numerous small mirrors may become feasible in space, using micro-thrusters for control, as demonstrated by the PRISMA test of the Swedish National Space Board with a pair of agile micro- satellites, controlled with centimeter accuracy. A version controlled by solar radiation pressure has been proposed to the European Space Agency (Labeyrie et al. 2009), and mentioned in the U.S. Decadal Survey as suitable for observing faint extra-galactic sources such as AGNs (Kraemer et al. 2010). Another version using inch-sized mirrors, each accurately controlled by radiation pressure within standing waves from a pair of counter-propagating laser beams, is described by Labeyrie et al. (2010). 
Such hypertelescope flotillas have been studied in preliminary detail for versions at the kilometer scale, the $100 \mathrm{~km}$ scale needed to resolve exo-planetary detail, and the $100000 \mathrm{~km}$ scale needed to resolve details of the Crab Pulsar. The latter version appears workable in terms of orbit control at the Earth-Sun Lagrangian L2 point, as analyzed by Infeld (2006), but needs rather large mirror elements, typically 6 or $8 \mathrm{~m}$, to efficiently collect their diffracted light at the combined focus. Such large mirrors may themselves be feasible in "laser-trapped" form (Labeyrie 1979) with a thin membrane, possibly made of diamond or graphene.

The prospect for such large dilute astronomical instruments emitting many laser beams raises the question of whether some advanced exo-civilizations, if they exist, may have built them before being capable of building Dyson spheres (2011). If so, the peculiar characteristics of their laser light emission, with periodically swept wavelength and potentially detectable with ELTs or hypertelescopes, may provide better signatures of extra-terrestrial intelligence than the infra-red excess heretofore searched by SETI programs for detecting Dyson spheres on stars.

\section{Conclusions and future work}

In the way of higher angular resolution at optical wavelengths, the potential of direct imaging with hypertelescopes, and induced sensitivity gain, has been confirmed by computer simulations, laboratory experiments and limited sky observations with miniature versions. The technical feasibility of large Earth-based versions, currently tested at Haute Provence and in the Ubaye range, becomes confirmed for hectometric aperture sizes, and kilometric sizes are now considered. Although images can be reconstructed with speckle interferometry techniques, the direct images obtainable with adaptive cophasing are a major goal.

The attainment of high limiting magnitudes appears feasible with modified forms of Laser Guide Star systems under development, thus greatly extending the scope of high angular resolution observing in astronomy. Also, image deconvolution techniques becoming developed for the hypertelescope case give encouraging results. These prospects suggest that a major breakthrough is possible in the way of high-resolution observing, extending to extra-galactic and cosmological sources. It appears to justify efforts toward building progressively larger instruments, especially considering the feasibility of expandable arrays not requiring the kind of major initial investment needed for the dome and mount of an ELT. In space, considerably larger apertures should become feasible in the form of mirror flotillas, eventually spanning up to perhaps $100000 \mathrm{~km}$. Such instruments would provide enough resolution to resolve the central body, believed to be a $20 \mathrm{~km}$ neutron star, of the Crab pulsar.

\section{References}

Aime, C., Lanteri, H., Diet, M., \& Carlotti, A., 2012, A\&A, 543A, 42A

Arnold, L., Labeyrie, A., Mourard, D., 1996, Adv. Space Res., 18, 49

Boccaletti, et al., 2000, Icarus, 145, 636 
Bonaccini Calia, D., Myers, R.M., Zappa, F., et al., 2004, SPIE, 5490, 1315

Borkowski, V., \& Labeyrie, A., 2004, EAS Publications Series, 12, 287

Buscher, D.F., Love, G.D., \& Myers, R.M., 2002, Opt. Lett., 27, 149

Bouyeron, L., Delage, L., Grossard, L., \& Reynaud, F., 2012, A\&A, 545, A18

Chapa, O., Cuevas, S., Sánchez, B., et al., 2007, Rev. Mex. Astron. Astrofis. Conf. Ser., 28,82

Crandall, R.S., et al., 1985, "Reversible optical storage medium and a method for recording information therein" US patent, http://www.google.com/patents/US4320489

Dainty, J.C., 1974, MNRAS, 169, 631

Dyson, F., 2011, see http://en.wikipedia.org/wiki/Dyson_sphere

Infeld, S.I., 2006, "Optimization of Mission Design for Constrained Libration Point Space Missions" Ph.D. Stanford, http://www.stanford.edu/group/SOL/dissertations/ samantha-thesis.pdf

Kraemer, S., Windhorst, R., Carpenter, K.G., et al., 2010, in "Astro2010: The Astronomy and Astrophysics Decadal Survey, Science White Papers, 162"

Le Coroller, H., Dejonghe, J., Arpesella, C., Vernet, D., \& Labeyrie, A., 2004, A\&A, 426, 721

Enmark, A., Andersen, T., Owner-Petersen, M., Chakraborty, R., \& Labeyrie, A., 2011, Integrated model of the Carlina Telescope", in "Integrated Modeling of Complex Optomechanical Systems", ed. Andersen, Torben, Enmark \& Anita, Proceedings of the SPIE, Vol. 8336, 83360J-83360J-14

Lardière, O., Martinache, F., \& Patru, F., 2007, MNRAS, 375, 977

Labeyrie, A., 1996, A\&A, 118, 517

Labeyrie, A., Le Coroller, H., \& Dejonghe, J., 2008, SPIE, 7013

Labeyrie, A., 2008, Proceedings of the SPIE, Vol. 6986, 69860C-69860C-12

Labeyrie, A., et al., 2009, Exper. Astron. 23, 463

Labeyrie, A., et al., 2010, "Resolved Imaging of Extra-Solar Photosynthesis Patches with a "Laser Driven Hypertelescope Flotilla", in "Pathways Towards Habitable Planets", proceedings of a workshop held 14 to 18 September 2009 in Barcelona, Spain, ed., Vincent Coudé du Foresto, Dawn M. Gelino \& Ignasi Ribas (San Francisco: Astronomical Society of the Pacific), 239

Labeyrie, A., et al., 2012, Optical and Infrared Interferometry III. Proceedings of the SPIE, Vol. 8445, id. 844512-844512-9

Labeyrie, A., et al., 2012, Optical and Infrared Interferometry III. Proceedings of the SPIE, Vol. 8445, id. 844511-844511-9

Labeyrie, A., 1979, A\&A, 77, L1

Labeyrie, A., Guillon, M., \& Fournier, J.M., 2012, "Optics of Laser Trapped Mirrors for large telescopes and hypertelescopes in space", SPIE conf.

Le Coroller, H., Dejonghe, J., Regal, X., et al., 2012, A\&A, 539, A59

Leger, et al., 2011, Astrobiology, 11, 4

Lohmann, A.W., Weigelt, G., \& Wirnitzer, B., 1983, Appl. Opt., 22, 4028

Martinache, F., 2004, J. Opt. A: Pure Appl. Opt., 6, 216

Martinache, F., 2012, A\&A, 286, 365

Mary, et al., 2013, EAS Publications Series, 59, 213 
Mavroidis, T., Solomon, C.J., \& Dainty, J.C., 1991, J. Opt. Soc. Am. A, 8, 1003

Mourard, D., et al., 2012, Optical and Infrared Interferometry III. Proceedings of the SPIE, Vol. 8445, id. 84451M-84451M-10

Patru, F., Tarmoul, N., Mourard, D., \& Lardière, O., 2009, MNRAS, 395, 2363

Patru, F., Chiavassa, A., Mourard, D., \& Tarmoul, N., Direct imaging with a hypertelescope of red supergiant stellar surfaces [eprint arXiv:1108.2320]

Pedretti, E., \& Labeyrie, A., 1999, A\&AS, 137, 543

Rabien, S., Eisenhauer, F., Genzel, R., Davies, R. I., \& Ott, T., 2006, A\&A, 450, 1, 2006, 415

Riaud, P., 2012, Eur. Phys. J. D, 66, 8

Surya, A., 2012, in preparation

Tcherniavski, I., 2011, Optical Engineering, 50, 3 